\title{
Politeness: A Relevant Issue for Relevance Theory*
}

\author{
Victoria Escandell-Vidal \\ UNED, Madrid
}

\begin{abstract}
The aim of this paper is to offer some guidelines for an explanation of politeness in the framework of Relevance Theory. I will suggest that the notion of social adequacy is to be preferred to that of strategy; as a primitive for politeness theory, and try to show how such a notion can be integrated into a cognitive model. Some aspects concerning the status of politeness will be then considered under the light of Wilson \& Sperber's (1993) distinctions: whether (or in which sense) it is an implicature, whether (or in which sense) it is communicated, whether (or in which sense) it is relevant. The predictions that emerge from adopting this point of view make it possible to provide a detailed and consistent account of politeness manoeuvres and effects.
\end{abstract}

\section{Introduction}

Politeness has become one of the fields of research to which more attention has been devoted in the last two decades. The connections of politeness studies with other domains, such as sociolinguistics and socio-pragmatics, ethnography of communication, second language teaching/acquisition or conversation analysis, have definitely contributed to this growing interest. Politeness theories and cognitive pragmatics, on the other hand, did not seem to have had an affinity for each other. ${ }^{1}$ This initial situation, however, has radically changed in the last few years, and several attempts to explain social aspects of communication from a cognitive perspective have been made. ${ }^{2}$ 
The aim of this paper is to contribute some ideas for this rapprochement by considering an explanation of politeness in the framework of Relevance Theory (hereinafter, RT), a model of verbal communication in which cognition plays a central role. I will begin by discussing what is politeness. Then, I will sketch the lines along which a picture can be drawn of politeness phenomena in relevance-theoretic terms. Finally, I will briefly comment on some consequences of adopting such an approach.

\section{On the nature of politeness}

\subsection{What is politeness?}

From social deixis to indirect speech acts, from conventional formulae to conversational strategies, from tact to friendliness, too many different things seem to have been bundled under a single label: indeed, politeness usually covers such a variety of phenomena that no consistent characterisation seems to be within reach.

In fact, in the standard meaning of the word polite at least three dimensions can be identified: 1) polite as civil or socially correct; 2) polite as kind or friendly; and 3) polite as tactful or diplomatic. A quick look at the literature easily shows that different researchers have favoured different senses. Echoing Fraser (1990), one could say that for Leech (1983) being polite involves making the hearer to 'feel good' (polite as friendly); to Brown \& Levinson (1987) it means making him not 'feel bad' (polite as diplomatic); ${ }^{3}$ for Fraser himself it is 'the expected state' (polite as socially correct). ${ }^{4}$

Although pointing in opposite directions, both Leech's and Brown \& Levinson's frameworks share a crucial property: they put the stress on the "functional" or strategic nature of politeness, ${ }^{5}$ against the 'old-fashioned' view that politeness is merely a set of arbitrary social conventions. Also the common distinction between 'positive politeness' and 'negative politeness' as the two sides of any politeness system emphasises the idea that the efforts made to show appreciation for the addressee or to avoid any intrusion into his legitimate privacy are the only constitutive parts of politeness.

However, a central issue seems to be missing from these approaches. Conversational strategies, ${ }^{6}$ or conversational efforts, can only have their raison d'être as exploitations of a default, 'unmarked' behaviour. Put in other words, being able to prevent undesirable results or to enhance positive effects entail having first a precise knowledge of expected courses of events (including, obviously, linguistic events and behaviour), and their social consequences. If so, politeness must primarily be a matter of social adequacy, established in terms of expectations. And any sort of conversational manoeuvres used to achieve a particular aim will be necessarily derived, since they will be dependent on strategic plans assuming default or expected effects. Thus, although analysing strategic moves is a significant part of a theory of politeness, only social adequacy can be a primitive notion.

Therefore, I am assuming an approach to politeness roughly along the lines of Fraser (1990:232): 
“... upon entering into a given conversation each party brings an understanding of some initial sets of rights and obligations that will determine, at least for the preliminary stages, what the participants can expect from the other(s)."

"The status, the power and the role of each speaker, and the nature of circumstances" are, according to Fraser (1990:232), the relevant parameters for calculating the initial set of rights and obligations. They "play a crucial role in determining what messages may be expected both in terms of form and content."

Status, power or social role are indeed basic notions to define social relationships. But there seems to be something unique to them and to social categorisation. Ordinary categorisation uses perception as its main source of data; social categorisation, by contrast, usually has to invoke elements and establish relationships with no direct perceptual motivation. Most of our social activities depend on created categories (such as profession, role or friendship), which are defined through social convention and do not necessarily relate to any essential or perceptible property of the individual. This fact strongly suggests that social cognition should be considered as a specific, separate domain or faculty. ${ }^{8}$

On the other hand, if defining factors depend ultimately on convention, a straightforward prediction will be that different cultures will presumably produce different created categories. Status, power, distance, social role or face, as abstract notions, are universal; but their particular content is clearly culture-sensitive': each society establishes its own conditions on what the relevant properties are that determine the values selected for each parameter. This explains why different cultures present radical differences in their politeness systems; and this explains also the misunderstandings in intercultural communication: what is polite (i.e., adequate) in a given culture may be seen as rude in other communities.

Politeness can be, thus, conceived of as a matter of social adequacy. Polite is the word we use to refer to a (linguistic) behaviour conforming to a given set of cultural norms: this is its central meaning; strategic uses of language should be parasitic on it. Only if a detailed characterisation is first provided of what counts as socially adequate can a proper account be offered for further-reaching manoeuvres.

\subsection{Politeness as social adequacy: How can it fit in RT?}

Now, if politeness is a matter of social adequacy and social adequacy is, in its turn, dependent on culture-specific norms, how can a psychological framework, such as RT, account for it? This question can be given, I think, a straightforward answer: if politeness is based on expectations, then it can be explained in terms of knowledge, ${ }^{10}$ if so, a psychological, cognitive framework will be able to account for it.

In the previous section, I have suggested that having expectations on courses of events and on their social consequences is necessary to account both for default and strategic politeness. But what are expectations? Expectations can be seen as a particular kind of mental (meta)representations, one that relates a state or an event to another state or event on the basis of a causal relationship previously attested the more an expectation is 
confirmed, the stronger it will become. The causal link can be natural or conventional: for instance, if I hold an apple in my hand and drop it, I expect that it will fall; similarly, if I do you a favour, I would probably expect that you expressed gratitude. The first expectation has a natural basis and belongs to naïve physics; the second one depends on convention and has to do with politeness.

Being mental representations, expectations are by definition a part of the individual's knowledge __ and since they are built and reinforced as the result of previous experience, they are a part of the individual's knowledge even in a stronger sense. However, it could be argued that this view is focusing on the individual side of expectations. What about their social side?

Sperber's (1996) theory of culture as epidemiology of representations offers a suitable framework for explaining the relationship between what is individual and what is social:

\begin{abstract}
"Consider a social group (...). Each member of the group has, in his or her head, millions of mental representations, some short-lived, others stored in long-term memory and constituting the individual's 'knowledge'. Of these mental representations, some _ _ a very small proportion __ get communicated repeatedly, and end up being distributed throughout the group, and thus have a mental version in most of its members. When we speak of cultural representations, we have in mind __ or should have in mind __ such widely distributed, lasting representations." (Sperber, 1996: 33)
\end{abstract}

Like other convention-based representations, expectations concerning social behaviour are not directly a result of examining "raw" facts, but rather of taking into account socially accepted interpretations (i.e., widely distributed metarepresentations) of facts. ${ }^{11}$ Therefore, they will have the form of "a higher-level representation [which] describes (...) a type of lower-level representation and the conditions under which versions of it can be produced and distributed." (Sperber, 1996: 30). As one can imagine, a number of social expectations will have to do with the use of certain linguistic expressions: those will contain a characterisation both of the expression and of the conditions under which it will be appropriate. $^{12}$

Being conventional and interpretive, the causal relationship cannot be merely inferred on the basis of regular patterns, but must be learned. As Janney \& Arndt (1992: 30) put it, "growing up to become a normal member of a culture is largely a matter of learning how to perceive, think, and behave as others in the culture do." The immediate prediction is that conventions may differ from culture to culture: cultural variation can thus be seen as the result of the distribution of different sets of representations concerning both the embedded representation and the conditions that determine its appropriate uses.

If seen as internal __ though widely distributed _ representations, expectations about linguistic behaviour fit easily in RT. Mental representations play indeed a major role in RT: the interpretation of any utterance involves, on the part of the hearer, the selection ${ }^{13}$ of a particular subset of his assumptions ${ }^{14}$ in order to derive contextual effects (in the form of newly derived assumptions or of strengthening or weakening of existing assumptions). However, assumptions are not totally independent from one another: in fact, information 
is usually stored as an organised set of related assumptions (Sperber \& Wilson, 1986/1995: 87-88). If so, expectations merely work in the regular way: they make more accessible some related set of assumptions, which provides a ready-made context for interpretation.

\section{On the status of politeness within RT}

The question of how RT can explain politeness effects involves a reconsideration of a wellknown problem: What is the status of politeness? Is it always an implicature? Does it always constitute a message?

Classical approaches to politeness those that see it as a conversational strategy seemed to have taken for granted that it is the result of an implicature, in which the length of the inferential path represents the degree of politeness. But if politeness is conceived basically as a matter of social adequacy and crucially depends on expectations (i.e., on knowledge), one could wonder whether this characterisation would still apply.

One of the main consequences of conceiving politeness effects as the result of an implicature is that it will always constitute a message and will be always communicated. The issue whether politeness is communicated has been recently addressed from a relevancetheoretic perspective by Jary in a most interesting paper (Jary, in press). According to his proposal, a hearer can find that some aspects of the speaker's verbal behaviour are compatible or incompatible with his assumptions (i.e., expectations) about the participants' relationship. If compatible, they "are not relevant enough to be worth the hearer's attention (=route (i))". If incompatible, they turn to be relevant enough to be worth the hearer's attention as evidence that the speaker holds him in higher or lower regard that he had assumed. Depending on whether he attributes intention to the speaker, four more possibilities of interpretation are obtained: Higher esteem, no attribution of intention = route (ii); higher esteem, plus attribution of intention = route (iii); lower esteem, no attribution of intention $=$ route $(i v)$; and lower esteem, plus attribution of intention $=$ route (v).

In Jary's proposal, the basic factors that explain the differences between all these routes of interpretation are the compatibility between expectations and actual linguistic behaviour on the one hand, and the attribution of intentionality to the speaker, on the other hand. Only in those cases in which the use of a linguistic form is seen as incompatible with expectations, and this incompatibility is seen as intentional by the hearer could it constitute a communicated assumption. Though I totally agree that those are indeed crucial factors, I am not as sure about the fact that (in)compatibility should totally determine the relevance of an assumption.

What I want to do in the next sections is to follow a slightly different path. I will begin by considering the relationship between politeness and relevance. Then, I will use the distinctions established in Wilson \& Sperber (1993) regarding different ways of carrying assumptions as a starting point for a characterisation of different politeness phenomena and effects. 


\subsection{Conveying vs. communicating assumptions}

The distinctions proposed in Wilson \& Sperber (1993) are central at this point. With every utterance a set of assumptions is made manifest. Presumably, some of these assumptions will be salient enough to be worth the hearer's while, so they will be processed and interpreted. However, not all the assumptions conveyed by an utterance __ not even those that are found relevant _ need to have been ostensively communicated.

For example, if you are a native speaker of Spanish, you could notice that I speak Spanish with a standard Madrid accent. Of course, this could perfectly go unnoticed as well. However, if you were to be asked about my accent, you could possibly remember what it was like. In addition, at a given time you could find it relevant for a number of different and unrelated reasons: if you are an Argentinian linguist looking for a speaker of European Spanish to test some data, this will constitute a convincing piece of evidence that I could be of some help to you; or you just could find the assumption that I am from Madrid strange, since it might contradict a previous assumption of yours that I am Catalan _ my family name is Catalan, so you could expect that I should have a Catalan accent instead. Both if you find the assumption that I am from Madrid relevant or not, you can conceivably guess that I am not intending to ostensively transmit it: it was not communicated by my utterance, but merely (unintentionally) conveyed by it. Thus only some of the assumptions that an utterance makes manifest are intentionally communicated.

Suppose now that $I$ want to get a role in a play for which an Argentinian actress is required, so I try and do my best to imitate an Argentinian accent. Although I am doing it intentionally, I probably would not intend that the cast director recognised that I am pretending. All my utterances will intentionally convey the (false) assumption that I am an Argentine, but the fulfilment of my purposes will require that $I$ do it in a covert way. The intention to transmit a certain assumption is not a sufficient condition either for saying that it has been communicated.

For real communication to take place, it is necessary that the transmission of assumptions is both intentional and overt, i.e., ostensive. Suppose now that I am speaking with my usual European Spanish accent and, at a given time, I begin to mimic an Argentinian accent; suppose also that, being a native speaker of Spanish, you can easily perceive and identify it. In this case, the change is both intentional and overt (mutually manifest), so it will call your attention as a stimulus carrying a communicated assumption. An expectation of relevance will arise according to which you will be encouraged to search not just for a relevant interpretation, but for an optimally relevant one. For instance, I might have used it to overtly mark an utterance as an echoic representation attributed to a common friend who is from Argentina. Only in this latter case have we an example of ostensive communication. 
3.2. Politeness, intentionality, and mutual manifestness

What I want to argue is that the distinction between these three forms of conveying information (unintentional, intentional-and-covert, and intentional-and-overt) can be extended to the understanding of politeness effects.

Take first the case in which a speaker uses a standard form that is perfectly adequate to the particular social situation. Suppose that, when being introduced to someone, the speaker utters How do you do? Since the form is standard, the fact that she used it would presumably go unnoticed, and the hearer would discard processing any associate assumption. ${ }^{15}$ Of course, if you would ask the hearer or a third party whether the speaker was polite, they could say she was indeed civil, socially correct. In some situations, the hearer could also find it relevant in some particular sense, and derive some conclusions: for instance, as mentioned before, if the speaker is a foreigner, the hearer can infer that she has mastered the social rules of his culture. In any event, the utterance does not communicate politeness, since there is no intention to do it on the part of the speaker.

Suppose that the speaker fails to produce an adequate form: suppose, for instance, that having been asked How are you?, she answered Praise to God. The occurrence of an unexpected stimulus _ one that contradicts a social expectation __ is usually a salient phenomenon and is very likely to call the hearer's attention. He could process it as conveying different assumptions: if the speaker is a foreigner, he could imagine that she does not know the usual conventions of English; or he could suppose that she is a missionary... No matter what the interpretation is, if the intention to communicate it is not overt, the inadequacy of the utterance would be, at most, manifest to the hearer, but not mutually manifest, so communication would not take place either.

On the other hand, if a speaker used a form that did not reflect the social situation according to her own understanding of it, but one that, she assumed, could suit the hearer's (or others') expectations, she would be in some sense pretending. ${ }^{16}$ Suppose she hates her interlocutor and has secretly decided not to speak to him anymore. Suppose also that at a particular time the speaker's job depends on the hearer, who is in a higher social position. In order to maintain her job, the speaker talks to her boss and even tries to be kind to him. In this kind of situation, her behaviour would be intentional; the existing mismatch, however, should necessarily be covert, so no particular message would have been communicated either. The speaker would obtain the intended effect as long as the hearer did not discover such a mismatch. ${ }^{17}$

Finally, if the speaker uses a form that contradicts previous assumptions on expected behaviour in an overt (mutually manifest) way, this will really constitute a genuine case of a communicated assumption, ${ }^{18}$ and it should presumably draw the hearer's attention. Imagine, for example, that a mother, after having called his son for dinner several times, utters something like Is his royal highness ready for dinner?. Both mother and son know that, given the nature of their relationship, this is not a standard way of address. Being overt, this mismatch not only calls for an interpretation, but also triggers the presumption 
that there should be an optimally relevant one. ${ }^{19}$ Only in this latter case can it be considered as a communicated assumption.

My point is, thus, that the sense in which it can be said that an utterance communicates politeness (or impoliteness) is a very restricted one: only when (im)politeness is both intentional and overt can it transmit a communicated assumption; only in this case it comes with a presumption of relevance, so the hearer should pay attention to it because it promises an optimal balance between processing effort and cognitive effects. Otherwise, it will be at most a conveyed assumption, but will never constitute a communicated message.

\subsection{Relevance and politeness}

The distinctions suggested in the previous section seem to raise a further issue: When is politeness relevant? Is it relevant only when it is ostensively communicated? In the relevance-theoretic framework, an assumption is relevant in a context when it has some contextual effects in that context (Sperber \& Wilson (1986/1995: 122). Thus, to yield contextual effects an assumption must enter an inferential process in which it combines with other assumptions.

I will examine two sorts of situations in which politeness can be relevant without having been communicated. Consider first the case of the evaluation of politeness, that is, the fact that a hearer, or a third party, can provide _ _ usually upon request _ an evaluation of the politeness of a particular sequence uttered in some given circumstances. What he will do to characterise an utterance as polite or impolite is to use some of the assumptions conveyed by it to construct a higher-level explicature, under which he may also embed other levels of explicature: for example, in certain situations an utterance like Get out! could give rise to a metarepresentation like The speaker asked me to leave (and he did it) in a very rude way. Consider now a different case, one in which the hearer spontaneously obtains some contextual effects. Suppose, for instance, that the use of a standard form by the speaker reinforces his previous assumption that she is learning quickly the conversational routines of his culture.

Though they both involve inferential processes, neither of these facts involves communication of politeness. It is true that, in both cases, the hearer brings into play some set of assumptions he draws from the utterance as premises for an inferential process. It is also true that they happen to be relevant, since some contextual effects can be obtained at no unnecessary processing cost by taking them into account. But what the examples above show is merely that any assumption obtained from any source can feed an inferential process. And in fact, as one would reasonably foresee, this sort of effects is not unique to politeness. Any phenomenon, be it linguistic or not, could produce similar results: from lexical choices to pronunciation, or from body language to voice quality, any of such aspects can give rise to relevant cognitive effects. ${ }^{20}$

If so, the same reasoning can apply to the cases in which the hearer feels there is a mismatch between his expectations and the speaker's linguistic behaviour. He can interpret 
the mismatch as strengthening his assumption that the speaker does not know how to behave properly; or as making manifest __ though not necessarily mutually manifest __ that she is being more polite than required by the situation. The 'failure' to produce a standard, default form may or may not be intentional on the side of the speaker. It can also be interpreted as intentional or not by the hearer. ${ }^{21}$ What is significant here is that it can be found relevant _ _ regardless of the fact of whether it is intentional or not __, and that relevance is thus independent from the speaker's intentionality: in the definition of relevance nothing is said about the source of assumptions, or the properties of the phenomenon from which they were obtained.

The fact that an assumption is used as a premise and found relevant does not entail that the assumption IS an implicature of the utterance __ at least, in the strong sense $\mathrm{e}^{22} \ldots$, nor does it entitle the hearer to suppose that the speaker wants to communicate this particular assumption. To properly qualify as an implicature in the strong sense an assumption must meet some other requirements, namely to have been both intentionally and ostensively communicated by the speaker.

From these considerations a conclusion can be drawn: when some property of the utterance is associated to some expectation involving social relationships, both fulfilling and contradicting expectations can be found relevant. ${ }^{23}$ The relative salience of the phenomenon (especially, its incompatibility with existing assumptions) will favour the possibility that it would be taken into account. ${ }^{24}$ However, it could never fully determine it.

A further prediction can be made. As suggested before, it is the overtness of a mismatch and not its mere existence what makes of it an instance of communication, and what carries a presumption of optimal relevance. If so, we can expect that also when an utterance fulfils the expectations, but does it in an overt way, some politeness effect will be obtained as well. This prediction is borne out. As Jary himself points out, if a teacher says to a class Could you please be quiet? overtly stressing the please, she is making mutually manifest "that she is behaving in accordance to the rules that govern their interaction and implicating that the pupils do the same." In fact, if we tend to think of (im)politeness effects as the result of a lack of correspondence between actual and expected behaviour it is only because we usually find it difficult to turn the use of a standard form into a stimulus with some overt salience, and not because there should be any a priori ban against such a possibility.

\section{Conclusion: Social adequacy, strategies and communication}

The considerations made in the previous sections have shown that the possibility of a strategic usage of linguistic forms with a social purpose is dependent on the existence of expectations about what can count as an adequate linguistic behaviour in a given situation. What is wrong with strategic approaches to politeness is not that strategic uses do not exist, but rather that all politeness phenomena are treated in terms of strategies: default uses are seen just as particular cases of strategies, and therefore all politeness effects are instances 
of implicature. As I have tried to suggest, this view misses two crucial distinctions: the one between intentional and non-intentional transmission of assumptions, and the one between overt and covert transmission of assumptions.

Here is where Relevance Theory can contribute to the understanding of politeness. The relevance-theoretic framework offers useful tools for establishing those distinctions and for deriving explanatory consequences from them: it is intentionality that turns a usage into a strategy; and it is mutual manifestness that turns a conveyed assumption into a communicated assumption. Any overt departure from the expected basis will communicate an assumption, but also any overt use of a standard form could produce a similar effect: only in these situations, in which politeness is communicated, can we talk about implicatures. However, the strategic exploitations of a standard behaviour do not reduce to the cases of communicated assumptions. Also when the speaker covertly intends to make the hearer feel that they are in a relationship closer that expected (or more distant that assumed) we are dealing with a kind of strategic use of politeness, although no implicature is being overtly transmitted.

On the side of the speaker intentionality is the key to distinguishing between the usage of a standard form (be it right or wrong) and the exploitation of expectations; by contrast, mutual manifestness is the notion that allows a distinction between merely conveying and actually communicating assumptions about social relationships. On the side of the hearer, only overtness guarantees the presumption of an optimally relevant interpretation. True, any phenomenon can draw the audience's attention and can be found relevant, no matter if it is adequate or not, no matter if it has been used intentionally or not; but the procedure itself is not ensured by ostensive communication. Any implication the hearer draws is obtained at his own risk. What is unique to real (ostensive) communication __ "the most important means by which the psychological tendency to maximise relevance is socially exploited" (Sperber \& Wilson, 1997: 149) is that is also communicates a presumption of (cognitive) optimality.

\section{Notes}

* I am very grateful to $\mathrm{M}$. Jary and $\mathrm{M}$. Leonetti for their comments on a first draft on this paper, and also to A. Auchlin, N. Franken, M. Jary and V. Zegarac for their bibliographical help. Needless to say, all possible mistakes are my own responsibility.

1. From the cognitive side, politeness phenomena and pragmatics have been considered as two very different perspectives, with no particular connection between them. "There is no conflict between Leech's socio-pragmatics approach and the present psychological approach, because they are attempting to do different things. For this reason it is misleading to include phenomena like politeness, face-saving and turn-taking together with the phenomena discussed in the following chapters under the general heading of pragmatics." (Blakemore, 1992:47).

2. See, for example, Jucker (1988), Clark [1994], Escandell-Vidal (1996), Jary (in press) and Jary (this volume), Zegarac (in press). 
3. Or "to make possible communication between potentially aggressive parties" (Brown and Levinson, 1987:1).

4. "Politeness is a state that one expects to exist in every conversation; participants note not that someone is being polite - this is the norm - but rather that the speaker is violating the Clonversational] Clontract]. Being polite does not involve making the hearer to 'feel good' à la Lakoff or Leech, nor making the hearer not 'feel bad' à la B[rown] \& L[evinson]. It simply involves getting on with the task at hand in light of the terms and conditions of the $\mathrm{CC}$. The intention to be polite is not signalled, it is not implicated by some deviation(s) from the most 'efficient' bald-on record way of using the language." (Fraser, 1990:233).

5. See Held (1992), Watts, Ide \& Ehlich (1992), or Werkhofer (1992), among others. In Escandell-Vidal (1996) I have presented a more detailed discussion of the points raised in this section.

6. In the studies on politeness the word strategy is sometimes used in a sense that clearly differs from its everyday meaning. When talking about 'politeness strategies' or 'discourse strategies', pragmaticians usually refer to different linguistic ways of conveying a certain speech act: for instance, using an imperative, posing a question on the hearer's possibility or willingness to comply an action, or pointing to an unwanted state of affairs can count as different request strategies. Since most of the times the speaker cannot really choose among the different ways, but is compelled by the situation to select only one of these possibilities, I find the use of the word strategy in these contexts rather misleading. My use of strategy, on the other hand, will follow the standard usage and involve notions such as 'long-term planning'.

7. This formulation is referred to as the 'conversational contract'. However, the use of the word 'contract' can be somewhat misleading, since it could suggest some previous, explicit or mandatory agreement between the two parties, which is not the case. Rather, it has more of a cognitive state than of a legal requirement. In fact, as Fraser (1990: 232) further explains, "During the course of time, or because of a change in the context, there is always the possibility for a renegotiation of the conversational contract: the two parties may readjust what rights and what obligations they hold towards each other".

8. Its main task will be that of developing a coherent picture of the self in society As Jackendoff (1992: 74) puts it, "each individual's participation in the culture must be supported by cognitive organisation in the individual's mind. (...) The way individuals are capable of acting out within a society depends on the way they are capable of internally representing the social context."

9. As several researchers have pointed out, Brown \& Levinson's (1987) notion of face must be relativised: though intended as universal, in their original formulation it covered basically Anglo-Saxon social behaviour, but it was inadequate to account even for other Western cultures, let alone Eastern or African ones. Nevertheless, I think that this concept is still useful as a general notion summarising what is desirable for self- and other's image.

10. In Escandell-Vidal (1996) I presented a more detailed discussion of this issue.

11. Roughly in the sense developed in Searle (1995).

12. The fact that an expectation has the form of a related pair might produce the illusion that it constitutes a rule __ and, in fact, the members of the group can perceive it as such. However, as Searle (1995: Ch. 1) points out, conventions differ from rules in that they are arbitrary, while rules are motivated. Zegarac (in press) also suggest a distinction between standardisation and conventionalisation. 
13. This task is guided by the search of relevance.

14. Assumptions are "conceptual representations treated by the individual as a representation of the actual world (as opposed to fictions or desires)." (Sperber \& Wilson, 1986/1995:2)

15. This case would correspond to Jary's (in press) route (i).

16. This will be the case of Machiavellian manipulation noted by Jary (in press), which would correspond to some cases of route (ii).

17. The reason why a speaker wants to conceal or not her intention depends on the extent to which the recognition of it would benefit her own plans. As Sperber (1994) points out, if you want to please someone, the recognition of your intention to please can reinforce the effect; but if you want to frighten somebody, the recognition of your intention will destroy the intended effect.

18. This corresponds to Jary's routes (ii) and (iv).

19. This presumption is not available in the previous cases, in which the assumption is not made mutually manifest.

20. See Sperber \& Wilson (1986/1995: 3.6)

21. It depends on the sort of interpretive strategy the hearer uses. See Sperber (1994) for details

22. For the distinction between strong and weak implicatures, see, Sperber \& Wilson (1986/1995: 4.4)

23. As Sperber \& Wilson (1997: 149) put it,

"For instance, non-ostensively produced evidence of deference or submission, or of expectations of deference or submission from others, is highly relevant and effective in maintaining or challenging power relationships." (Sperber \& Wilson, 1997: 149)

24. "Even in these non-ostensive cases, considerations of cognitive relevance determine the degree of manifestness achieved, and the likelihood that the information will be picked up, and therefore play a role in further interaction." (Sperber \& Wilson, 1997: 149)

\section{Works Cited}

Blakemore, D. Understanding Utterances, Oxford, Blackwell, 1992.

Brown, P. and S. Levinson. Politeness. Some Universals in Language Use. Cambridge. Cambridge University Press, 1987.

Clark, B. "Social function of language: Insults and politeness." Paper presented at the LAGB Meeting (September 1994), Middlesex University, 1994.

Escandell-Vidal, V. "Towards a cognitive approach to politeness." Contrastive Semantics and Pragmatics (Vol. II: Discourse Strategies). Eds. K. Jaszczolt and K. Turner. Oxford: Pergamon, 1996. 629-650 (Also in Language Sciences, 18, pp. 629-650).

Fraser, B. "Perspectives on politeness." Journal of Pragmatics 14 (1990): 219-236.

Held, G. "Politeness in linguistic research." Politeness in Language. Studies in its History, Theory and Practice. Eds. R.J. Watts, S. Ide, and K. Ehlich. Berlin: Mouton-De Gruyter, 1992. 131-153.

Jackendoff, R. Languages of the Mind, Cambridge (Mass.), MIT Press, 1992.

Janney, R.W. and H. Arndt. "Intracultural tact vs intercultural tact." Politeness in Language. Studies in its History, Theory and Practice. Eds. R.J. Watts, S. Ide, and K. Ehlich. Berlin: 
Mouton-De Gruyter, 1992. 21-41.

Jary, M. (this volume): "Is relevance theory asocial?"

. "Relevance theory and the communication of politeness", Journal of Pragmatics 30 (1998): $1-19$.

Jucker, A.H. "The relevance of politeness." Multilingua 7 (1988): 375-384.

Lakoff, R. "The logic of politeness, or minding your p's and q's", Proceedings of the Ninth Regional Meeting of the Chicago Linguistic Society, 1973. 345-356.

Leech, G. Principles of Pragmatics, London: Longman, 1983.

Searle, J.R. The Construction of Social Reality, New York: Simon \& Schuster, 1995.

Sperber, D. "Undestanding verbal understanding." What Is Intelligence? Ed. J. Khalfa.

Cambridge: Cambridge University Press, 1994. 179-198.

Sperber, D. Explaining Culture. Oxford: Blackwell, 1996.

Sperber, D. and D. Wilson. Relevance: Communication and Cognition. Oxford: Blackwell, $1986 / 95$.

. "Remarks on relevance theory and the social sciences." Multilingua 16 (1997): 145151.

Werkhofer, K.T. "Traditional and modern views: The social constitution and the power of politeness." Politeness in Language. Studies in its History, Theory and Practice. Eds. R.J. Watts, S. Ide, and K. Ehlich. Berlin: Mouton-De Gruyter, 1992. 155-199.

Wilson, D. and D. Sperber. "Linguistic form and relevance." Lingua 90 (1993): 1-25.

Zegarac, V. "What is phatic communication?" Current Issues in Relevance Theory. Eds.V. Rouchota and A.H. Jucker. Amsterdam, John Benjamins, in press. 